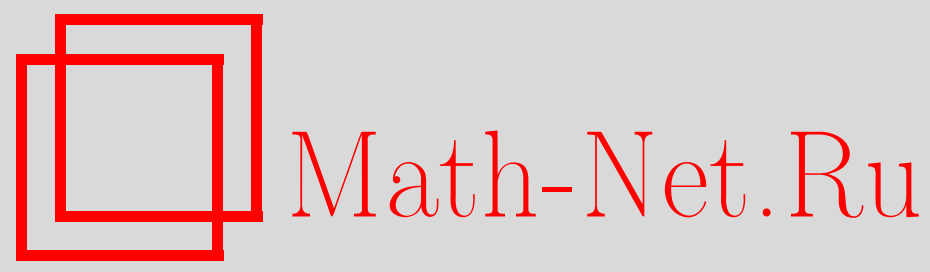

А. И. Столяров, О. Е. Тихонов, А. Н. Шерстнев, Характеризация нормальных следов на алгебрах фон Неймана неравенствами для модуля, Матем. заметки, 2002, том 72, выпуск 3, 448-454

DOI: https://doi.org/10.4213/mzm435

Использование Общероссийского математического портала Math-Net.Ru подразумевает, что вы прочитали и согласны с пользовательским соглашением http://www.mathnet.ru/rus/agreement

Параметры загрузки:

IP : 18.234 .156 .22

26 апреля 2023 г., $17: 22: 34$

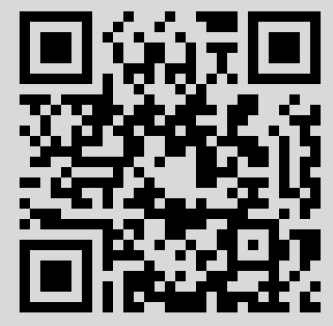




\title{
ХАРАКТЕРИЗАЦИЯ НОРМАЛЬНЫХ СЛЕДОВ НА АЛГЕБРАХ ФОН НЕЙМАНА НЕРАВЕНСТВАМИ ДЛЯ МОДУЛЯ
}

\author{
А. И. Столяров, О.Е. Тихонов, А.Н. Шерстнев
}

\begin{abstract}
Доказано, что если для нормального полуконечного веса $\varphi$ на алгебре фон Неймана $\mathscr{M}$ для любых самосопряженных операторов $a_{1}, a_{2}$ из $\mathscr{M}$ выполняется неравенство $\varphi\left(\left|a_{1}+a_{2}\right|\right) \leqslant \varphi\left(\left|a_{1}\right|\right)+\varphi\left(\left|a_{2}\right|\right)$, то этот вес является следом. Приведен ряд других схожих характеризаций следов среди нормальных полуконечных весов. В частности, уточнен и усилен результат Л. Т. Гарднера о характеризации следов неравенством $|\varphi(a)| \leqslant \varphi(|a|)$.

Библиография: 14 названий.
\end{abstract}

Хорошо известно (см., например, $[1$, гл. $1, \S 6$, п. 10]), что для произвольного следа $\varphi$ на алгебре фон Неймана $\mathscr{M}$ и любых операторов $a_{1}, a_{2} \in \mathscr{M}$ выполняется неравенство

$$
\varphi\left(\left|a_{1}+a_{2}\right|\right) \leqslant \varphi\left(\left|a_{1}\right|\right)+\varphi\left(\left|a_{2}\right|\right) .
$$

Пусть $\varphi$ - нормальньй полуконечный вес на $\mathscr{M}$ такой, что это неравенство вьполняется для любых самосопряженных операторов из $\mathscr{M}$. Основная цель настоящей заметки показать, что тогда рассматриваемый вес является следом. Насколько известно авторам, характеризация следа такого типа не упоминалась в литературе даже для случая положительных функционалов на алгебре $(2 \times 2)$-матриц.

1. Обозначения, определения и предварительные сведения. Для алгебры фон Неймана $\mathscr{M}$ операторов в гильбертовом пространстве через $\mathscr{M}^{\mathrm{sa}}, \mathscr{M}^{+}{ }_{\text {и }} \mathscr{M}^{\mathrm{pr}}$ будем обозначать ее подмножества самосопряженньх операторов, положительных операторов и проекторов соответственно, $\mathscr{M}^{\mathrm{s}}$ - совокупность симметрий, т.е. унитарных самосопряженных операторов из $\mathscr{M}, e$ - единица алгебры $\mathscr{M}$.

ОПредЕлЕниЕ 1. Весом на алгебре фон Неймана $\mathscr{M}$ назьвается отображение $\varphi$ : $\mathscr{M}^{+} \rightarrow[0,+\infty]$ со свойствами

$$
\varphi(x+y)=\varphi(x)+\varphi(y), \quad \varphi(\lambda x)=\lambda \varphi(x), \quad x, y \in \mathscr{M}^{+}, \quad \lambda \geqslant 0
$$

(при этом $0 \cdot(+\infty) \equiv 0)$.

Для веса $\varphi$ на $\mathscr{M}$ через $\mathfrak{m}_{\varphi}^{+}$будем обозначать множество $\left\{x \in \mathscr{M}^{+} \mid \varphi(x)<+\infty\right\}$, через $\mathfrak{m}_{\varphi}-$ его комплексную линейную оболочку. Множество $\mathscr{M}^{\mathrm{pr}} \cap \mathfrak{m}_{\varphi}^{+}$проекторов с конечным весом будем обозначать через $\mathfrak{p}_{\varphi}$.

Работа выполнена при частичной поддержке Российского фонда фундаментальных исследований, грант № 01-01-00129, и научной программы "Университеты России - фундаментальные исследования", грант № 990213 . 
ОПРЕДЕЛЕНиЕ 2. Вес $\varphi$ на алгебре фон Неймана $\mathscr{M}$ назьвается

- конечным, если $\varphi(e)<+\infty$;

- нормальным, если $\sup \varphi\left(x_{i}\right)=\varphi\left(\sup x_{i}\right)$ для любой монотонно возрастающей ограниченной сети $\left\{x_{i}\right\} \subset \mathscr{M}^{+}$;

- полуконечным, если множество $\mathfrak{m}_{\varphi}$ ультраслабо плотно в $\mathscr{M}$;

- следом, если $\varphi\left(x^{*} x\right)=\varphi\left(x x^{*}\right)$ для любого $x \in \mathscr{M}$.

Вес $\varphi$ однозначно продолжается до линейного функционала на $\mathfrak{m}_{\varphi}$, обозначаемого обычно той же буквой $\varphi$. В частности, конечньй вес однозначно продолжается до положительного функционала на $\mathscr{M}$. Это позволяет отождествлять конечные веса и положительные функционалы на $\mathscr{M}$.

В работе [2] показано, что условие нормальности веса $\varphi$ эквивалентно существованию некоторого множества $F$ нормальных положительных функционалов на $\mathscr{M}$ такого, что $\varphi(x)=\sup _{\omega \in F} \omega(x)$ для любого $x \in \mathscr{M}^{+}$. Это позволяет нам при исследовании нормальных весов использовать результаты работы [3].

Хорошо известно, что вес $\varphi$ на $\mathscr{M}$ является следом тогда и только тогда, когда $\varphi(x)=$ $\varphi\left(u x u^{*}\right)$ для любого $x \in \mathscr{M}^{+}$и любого унитарного оператора $u \in \mathscr{M}$. При доказательстве следующей характеризации следов существенно используются результаты Х. Упмайера [4].

ПРЕДЛОЖЕНИЕ 1 (см. [5, теорема I.4.2]). Вес $\varphi$ на алгебре фон Неймана $\mathscr{M}$ является следом тогда и только тогда, когда $\varphi(x)=\varphi(s x s)$ для любых $x \in \mathscr{M}^{+} u$ $s \in \mathscr{M}^{\mathrm{s}}$.

ПРЕДЛОЖЕНИЕ 2. Пусть $\varphi-$ нормальный полуконечный вес на полуконечной алгебре фон Неймана $\mathscr{M}$. Тогда существует семейство попарно ортогональных проекторов из $\mathfrak{p}_{\varphi}$, в сумме дающих е.

ДоКАЗАТЕЛЬСТВО. Пусть $\tau$ - точный нормальный полуконечный след на $\mathscr{M}$ (существование такого следа гарантируется полуконечностью алгебры). Согласно теореме 5.12 из [3] существует единственньй самосопряженньй положительньй оператор $h$, присоединенньй к $\mathscr{M}$, такой, что $\varphi=\tau(h \cdot)$. Пусть $p_{n}-$ спектральный проектор оператора $h$, соответствующий полуоткрытому интервалу $[n-1, n)$. Так как след $\tau$ полуконечен, то для каждого натурального $n$ найдется семейство $\left\{q_{i}\right\}_{i \in \Im_{n}}$ попарно ортогональных проекторов из $\mathfrak{p}_{\tau}$ такое, что $p_{n}=\sum_{i \in \Im_{n}} q_{i}$. Из ограниченности операторов $h p_{n}$ следует, что все $q_{i}$ из $\mathfrak{p}_{\varphi}$. Таким образом,

$$
e=\sum_{i \in \bigcup_{n=1}^{\infty} \Im_{n}} q_{i}
$$

- требуемое представление $е$ в виде суммы попарно ортогональных проекторов из $\mathfrak{p}_{\varphi}$.

Для алгебры фон Неймана $\mathscr{M}$, веса $\varphi$ на ней и проектора $p$ из $\mathscr{M}$ через $\mathscr{M}_{p}$ будем обозначать соответствующую редуцированную алгебру фон Неймана (см. [1, гл. $1, \S 2$, п. 1]), через $\varphi_{p}-$ редуцированный вес на $\mathscr{M}_{p}$, получающийся из ограничения на $p \mathscr{M} p$ веса $\varphi$. Заметим, что если $\varphi$ нормален и $p \in \mathfrak{p}_{\varphi}$, то $\varphi_{p}-$ нормальный конечный вес на $\mathscr{M}_{p}$. 


\section{2. Характеризация следов неравенством субаддитивности для модуля.}

ЛЕмма 1. Пусть для веса $\varphi$ на алгебре фон Неймана $\mathscr{M}$ и некоторых операторов $а \in \mathscr{M}^{+}, s \in \mathscr{M}^{\mathrm{s}}$ выполняются неравенства $\varphi($ sаs $)<\varphi(a)<\infty$. Тогда найдутся операторы $b_{1}, b_{2} \in \mathscr{M}^{+}$такие, что $\varphi\left(\left|b_{1}-b_{2}\right|\right)>\varphi\left(b_{1}\right)+\varphi\left(b_{2}\right)$.

ДоКАЗАТЕЛЬСТво. Обозначим $\varepsilon=(\varphi(a)-\varphi(s a s)) / \varphi(a)$ и для параметра $\lambda>0$ рассмотрим операторы $b_{1}(\lambda)=\lambda s a s+a s+s a+\lambda^{-1} a, b_{2}(\lambda)=\lambda s a s-a s-s a+\lambda^{-1} a$. При любом $\lambda>0$ эти операторы положительны (так как, например, $b_{1}(\lambda)=\lambda\left(s+\lambda^{-1} e\right) \times$ $\left.a\left(s+\lambda^{-1} e\right)\right)$ и $\varphi\left(b_{1}(\lambda)\right)+\varphi\left(b_{2}(\lambda)\right)=2(\lambda(1-\varepsilon)+\lambda) \varphi(a)$. Заметим, что

$$
\inf _{\lambda>0}\left\{\lambda(1-\varepsilon)+\lambda^{-1}\right\}=2(1-\varepsilon)^{1 / 2} .
$$

Непосредственными вычислениями проверяется, что $\left|b_{1}(\lambda)-b_{2}(\lambda)\right|=2(s a s+a)$ и $\varphi\left(\left|b_{1}(\lambda)-b_{2}(\lambda)\right|\right)=2(2-\varepsilon) \varphi(a)$. Так как $(2-\varepsilon)>2(1-\varepsilon)^{1 / 2}$, за счет выбора подходящего $\lambda$ можно получить желаемые операторы $b_{1}$ и $b_{2}$.

Из предложения 1 и леммы 1 непосредственно вытекает следующая характеризация конечных следов.

ТеОремА 1. Пусть $\varphi-$ конечный вес на алгебре фон Неймана $\mathscr{M}$. Если для любых операторов $a_{1}, a_{2} \in \mathscr{M}^{\mathrm{sa}}$ выполняется неравенство (*), то $\varphi-$ след.

ЗАмЕчаниЕ 1. Другое доказательство теоремы 1 можно получить, проверив сначала ее справедливость для случая алгебры $M_{2}(\mathbb{C})$ комплексных $(2 \times 2)$-матриц, а затем воспользовавшись техникой работы [6].

Перейдем теперь к бесконечньм весам.

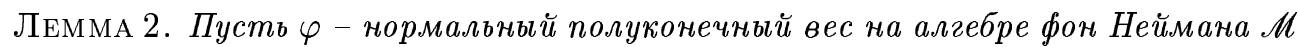
и для любого проектора $p \in \mathfrak{p}_{\varphi}$ редуцированный вес $\varphi_{p}$ на редуиированной алгебре

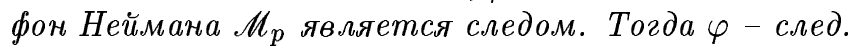

ДокАЗАТЕЛьСтво. Произвольная алгебра фон Неймана канонически изоморфна произведению полуконечной и чисто бесконечной алгебр фон Неймана (см., например, $[1$, гл. $1, \S 6$, п. 7]) и, как нетрудно убедиться, достаточно доказать рассматриваемую лемму для алгебр этих двух типов.

Пусть $\mathscr{M}$ - полуконечная алгебра фон Неймана. Из предложения 2 следует, что найдется монотонно возрастающая сеть $\left\{p_{\alpha}\right\}$ проекторов из $\mathfrak{p}_{\varphi}$, ультрасильно сходящаяся к $е$. Рассмотрим инволютивную подалгебру $\mathscr{A}=\bigcup_{\alpha} p_{\alpha} \mathscr{M} p_{\alpha}$ и конечный положительный функционал $\dot{\varphi}$ на ней, получаюшийся ограничением на $\mathscr{A}^{+}=\mathscr{A} \cap \mathscr{M}^{+}$веса $\varphi$ и продолжением по линейности этого ограничения на всю $\mathscr{A}$. Подалгебра $\mathscr{A}$ ультраслабо плотна в $\mathscr{M}, \dot{\varphi}\left(x^{*} x\right)=\dot{\varphi}\left(x x^{*}\right)$ для любого $x \in \mathscr{A}$ и функционал $y \mapsto \dot{\varphi}\left(x^{*} y x\right)$ ультраслабо непрерьвен на $\mathscr{A}$ для любого $x \in \mathscr{A}$, поэтому согласно предложению 3.6 из [7] существует единственный нормальньй полуконечньй след на $\mathscr{M}^{+}$, совпадающий с $\dot{\varphi}$ на $\mathscr{A}^{+}$. Так как этот след совпадает с $\varphi$ на $\mathscr{A}^{+}$, то согласно предложению 3.3 из [7] он совпадает с $\varphi$ на всем $\mathscr{M}^{+}$, т.е. вес $\varphi$ является следом.

Если алгебра $\mathscr{M}$ чисто бесконечна, то любая редуцированная алгебра $\mathscr{M}_{p}$ также чисто бесконечна (см. $[1$, гл. $1, \S 6$, п. 8 , следствие 4 , с. 104]). Согласно предположениям леммы для любого $p \in \mathfrak{p}_{\varphi}$ вес $\varphi_{p}$ на $\mathscr{M}_{p}$ является (нормальным, конечным) следом, нулевым в силу чистой бесконечности $\mathscr{M}_{p}$. Таким образом, $\varphi(p)=0$ для любого $p \in \mathfrak{p}_{\varphi}$ и из полуконечности веса $\varphi$ следует, что он нулевой. Это заканчивает доказательство леммы. 
ТЕОРемА 2. Пусть $\varphi$ - нормальный полуконечный вес на алгебре фон Неймана $\mathscr{M}$. Если для любых операторов $a_{1}, a_{2} \in \mathscr{M}^{\mathrm{sa}}$ выполняется неравенство $(*)$, то $\varphi-$ след.

ДокАЗАТЕЛЬСтво. Ясно, что при любом $p \in \mathfrak{p}_{\varphi}$ для любых $a_{1}, a_{2} \in \mathscr{M}_{p}^{\text {sa }}$ вьполняется неравенство $\varphi_{p}\left(\left|a_{1}+a_{2}\right|\right) \leqslant \varphi_{p}\left(\left|a_{1}\right|\right)+\varphi_{p}\left(\left|a_{2}\right|\right)$, поэтому согласно теореме 1 конечньй вес $\varphi_{p}$ на $\mathscr{M}_{p}$ является следом. По лемме $2 \varphi$-след.

3. Описание нормальных весов, удовлетворяющих неравенству субаддитивности для модуля. Для веса $\varphi$ на алгебре фон Неймана $\mathscr{M}$ через $q(\varphi)$ будем обозначать проектор из $\mathscr{M}$ такой, что ультраслабое замыкание $\mathfrak{m}_{\varphi}$ есть $q(\varphi) \mathscr{M} q(\varphi)$ (см. [7, $\S 2])$. Ясно, что вес $\varphi_{q(\varphi)}$ полуконечен.

Пусть $p \in \mathscr{M}^{\mathrm{pr}}$. Для $a \in \mathscr{M}^{+}$положим $\theta^{(p)}(a)=0$, если $a=p a p$, и $\theta^{(p)}(a)=+\infty$ в противном случае. Нетрудно видеть, что $\theta^{(p)}$ - нормальньй вес на $\mathscr{M}$ и $q\left(\theta^{(p)}\right)=p$.

ТЕОремА 3. Для нормального веса $\varphi$ на алгебре фон Неймана $\mathscr{M}$ следующие условия эквивалентны:

(i) для любых операторов $a_{1}, a_{2} \in \mathscr{M}$ выполняется неравенство (*);

(ii) для любых операторов $a_{1}, a_{2} \in \mathscr{M}^{\text {sa }}$ выполняется неравенство (*);

(iii) для любого проектора $p \in \mathfrak{p}_{\varphi}$ вес $\varphi_{p}$ на $\mathscr{M}_{p}$ является следом;

(iv) вес $\varphi_{q(\varphi)}$ на $\mathscr{M}_{q(\varphi)}$ является следом;

(v) вес $\varphi$ представляется в виде суммы $\varphi=\tau+\theta^{(p)}$, где $\tau-$ нормальный полуконечный след на $\mathscr{M}$, а $р$ - некоторый проектор из $\mathscr{M}$.

ДокАЗАТЕЛьСтво. Справедливость импликации (i) $\Longrightarrow$ (ii) очевидна. Для доказательства импликации (ii) $\Longrightarrow$ (iii) достаточно повторить первую фразу из доказательства теоремы 2. Справедливость импликации (iii) $\Longrightarrow$ (iv) следует из леммы 2.

Докажем импликацию (iv) $\Longrightarrow(\mathrm{v})$. Пусть $\varphi$ - нормальньй вес, удовлетворяюший условию (iv), и пусть $c(q(\varphi))$ обозначает центральный носитель проектора $q(\varphi)$. Согласно [8, лемма 2] нормальньй полуконечньй след $\varphi_{q(\varphi)}$ на $\mathscr{M}_{q(\varphi)}$ продолжается до нормального полуконечного следа $\widetilde{\tau}$ на $\mathscr{M}_{c(q(\varphi))}$. (Требование точности рассматриваемого следа, имеющееся в [8], конечно, является несущественным.) Формула $\tau(a)=$ $\widetilde{\tau}(c(q(\varphi)) a)\left(a \in \mathscr{M}^{+}\right)$задает нормальньй полуконечньй след $\tau$ на $\mathscr{M}$. Из построения ясно, что $\varphi=\tau+\theta^{(q(p))}$.

Убедимся, наконец, в справедливости импликации (v) $\Longrightarrow$ (i). Достаточно доказать, что для любых $a_{1}, a_{2} \in \mathscr{M}, p \in \mathscr{M}^{\mathrm{pr}}$ выполняется неравенство субадлитивности $\theta^{(p)}\left(\left|a_{1}+a_{2}\right|\right) \leqslant \theta^{(p)}\left(\left|a_{1}\right|\right)+\theta^{(p)}\left(\left|a_{2}\right|\right)$, а для этого достаточно показать, что условие $\theta^{(p)}\left(\left|a_{1}\right|\right)=\theta^{(p)}\left(\left|a_{2}\right|\right)=0$ влечет $\theta^{(p)}\left(\left|a_{1}+a_{2}\right|\right)=0$. В справедливости же последнего утверждения нетрудно убедиться, проверив, что для $a \in \mathscr{M}$ и $p \in \mathscr{M}^{\mathrm{pr}}$ условие $\theta^{(p)}(|a|)=0$ эквивалентно условию $\theta^{(p)}\left(|a|^{2}\right)=0$, т.е. условию $a^{*} a=p a^{*} a p$, что, в свою очередь, эквивалентно условию $a(e-p)=0$.

ЗАмЕчАниЕ 2. Нетрудно проверить, что вес $\theta^{(p)}$ является следом тогда и только тогда, когда проектор $p$ содержится в центре алгебры $\mathscr{M}$. Отсюда видно, что условие полуконечности в теореме 2 отбросить нельзя. 
4. Некоторые другие характеризации следов неравенствами. Тривиальным образом проверяется, что для произвольного веса $\varphi$ на алгебре фон Неймана $\mathscr{M}$ условие (ii) теоремы 3 эквивалентно следующему условию вьпуклости для модуля:

(vi) для любых операторов $a_{1}, a_{2} \in \mathscr{M}^{\mathrm{sa}}$ илюбого $\alpha \in[0,1]$ выполняется неравенство

$$
\varphi\left(\left|\alpha a_{1}+(1-\alpha) a_{2}\right|\right) \leqslant \alpha \varphi\left(\left|a_{1}\right|\right)+(1-\alpha) \varphi\left(\left|a_{2}\right|\right) .
$$

ПРЕДЛОЖЕНИЕ 3. Для произвольного веса $\varphi$ на алгебре фон Неймана М условие (ii) теоремы 3 әквивалентно следующему условию:

(vii) для любого оператора $a \in \mathscr{M}^{\mathrm{sa}}$ выполняется соотношение

$$
\varphi(|a|)=\inf \left\{\varphi\left(a^{\prime}\right)+\varphi\left(a^{\prime \prime}\right) \mid a^{\prime}, a^{\prime \prime} \in \mathscr{M}^{+}, a=a^{\prime}-a^{\prime \prime}\right\} .
$$

ДоКАЗАТЕЛЬСТВо. Обозначим через $\delta_{\varphi}(a)$ правую часть равенства в условии (vii), и пусть $a=a^{+}-a^{-}$- разложение самосопряженного оператора в виде разности его положительной и отрицательной частей. Так как $\varphi(|a|)=\varphi\left(a^{+}\right)+\varphi\left(a^{-}\right)\left(a \in \mathscr{M}^{\text {sa }}\right)$, то для любого веса $\varphi$ и любого $a \in \mathscr{M}^{\text {sa }}$ выполняется неравенство $\delta_{\varphi}(a) \leqslant \varphi(|a|)$.

Предположим, что для веса $\varphi$ условие (vii) не выполнено. Тогда найдется оператор $a \in \mathscr{M}^{\text {sa }}$ такой, что $\delta_{\varphi}(a)<\varphi(|a|)$. Следовательно, найдется разложение $a=a^{\prime}-a^{\prime \prime}$ $\left(a^{\prime}, a^{\prime \prime} \in \mathscr{M}^{+}\right)$такое, что $\varphi\left(a^{\prime}\right)+\varphi\left(a^{\prime \prime}\right)<\varphi(|a|)$, т.е. $\varphi\left(\left|a^{\prime}+\left(-a^{\prime \prime}\right)\right|\right)>\varphi\left(\left|a^{\prime}\right|\right)+\varphi\left(\left|-a^{\prime \prime}\right|\right)$. Таким образом, показано, что для веса $\varphi$ не выполнено условие (ii), и доказана импликация (ii) $\Longrightarrow$ (vii).

Чтобы убедиться в справедливости импликации (ii) $\Longrightarrow$ (vii), достаточно заметить, что всегда вьполняется следующее "неравенство треугольника" :

$$
\delta_{\varphi}\left(a_{1}+a_{2}\right) \leqslant \delta_{\varphi}\left(a_{1}\right)+\delta_{\varphi}\left(a_{2}\right) \quad\left(a_{1}, a_{2} \in \mathscr{M}^{\mathrm{sa}}\right) .
$$

Таким образом, вьполнение любого из условий (vi), (vii) характеризует следы среди всех нормальных полуконечных весов.

Отметим, что выражение, стоящее в правой части равенства в условии (vii), существенно используется при одном из возможных подходов к построению пространства $L_{1}$, ассоциированного с нормальньм полуконечньм весом [9] (см. также [10]).

В работе [11] Л. Т. Гарднер для веса $\varphi$ на алгебре фон Неймана $\mathscr{M}$ рассматривал следующее условие:

(viii) Әля любого проектора $p \in \mathfrak{p}_{\varphi}$ для любого оператора $а \in \mathscr{M}_{p}$ выполняется неравенство

$$
\left|\varphi_{p}(a)\right| \leqslant \varphi_{p}(|a|)
$$

и доказал [11, теорема 2], что выполнение этого условия характеризует следы в классе нормальных “сильно полуконечных" весов, т.е. нормальных весов $\varphi$ на алгебре фон Неймана $\mathscr{M}$, удовлетворяющих требованию: для любого ненулевого оператора из $\mathscr{M}^{+}$ найдется мажорируемьй им ненулевой оператор из $\mathfrak{m}_{\varphi}^{+}$. (Такие веса, называя их “локально конечньми" , изучал также Н. В. Трунов [12]-[14].) Из результатов Гарднера [11, теорема 1] следует, однако, что выполнение для веса $\varphi$ условия (viii) влечет вьполнение для этого веса условия (iii) в теореме 3. (Обратная импликация, (iii) $\Longrightarrow$ (viii), хорошо известна.) Таким образом, получаем следующее уточнение и усиление одного из основных результатов работы [11]. 
ТЕОремА 4. Для нормального веса $\varphi$ на алгебре фон Неймана $\mathscr{M}$ выполнение условия (viii) равносильно выполнению каждого из приведенных выше условий (i)(vii). В частности, нормальный полуконечный вес $\varphi$, для которого выполняется условие (viii), является следом.

СлЕДСТВИЕ 1. Для нормального веса $\varphi$ на алгебре фон Неймана $\mathscr{M}$ выполнение каждого из приведенных выше условий (i)-(viii) равносильно выполнению условия

(ix) для любого оператора $a \in \mathfrak{m}_{\varphi}$

$$
|\varphi(a)| \leqslant \varphi(|a|)
$$

В частности, нормальный полуконечный вес $\varphi$, для которого выполняется условие (iх), является следом.

ДокАЗАТЕЛЬСтво. Ясно, что вьполнение условия (ix) влечет выполнение (viii), что влечет согласно теореме 4 выполнение, в частности, условия (iv). C другой стороны, как хорошо известно, условие (ix) вьполняется для любого следа $\varphi$. Отсюда, предположив, что для веса $\varphi$ выполнено условие (iv), учитьвая соотношение $\mathfrak{m}_{\varphi} \subset q(\varphi) \mathscr{M} q(\varphi)$, нетрудно заключить, что для $\varphi$ вьполнено и условие (ix).

СлЕдСТВИЕ 2. Нормальный вес $\varphi$ на алгебре фон Неймана М является следом тогда и только тогда, когда он обладает свойством

(х) для $a \in \mathscr{M}$ условие $\varphi(|a|)<\infty$ влечет $a \in \mathfrak{m}_{\varphi} u$

$$
|\varphi(a)| \leqslant \varphi(|a|)
$$

ДокАЗАТЕЛЬСТвО. То, что в случае, когда вес $\varphi$ является следом, он обладает свойством (х), хорошо известно.

Для нормального веса $\varphi$, обладающего свойством (х), очевидно, выполняется условие (viii), поэтому по теореме 4 вес $\varphi_{q(\varphi)}$ на $\mathscr{M}_{q(\varphi)}$ является следом. Как нетрудно убедиться, вес $\varphi$, для которого $\varphi_{q(\varphi)}-$ след, является следом тогда и только тогда, когда проектор $q(\varphi)$ центральньй, т.е. совпадает со своим центральньм носителем $c(q(\varphi))$.

Пусть нормальньй вес $\varphi$ обладает свойством (х). Предположим, что он не является следом. Тогда $c(q(\varphi))-q(\varphi) \neq 0$ и в $\mathscr{M}$ найдется ненулевой частично изометрический оператор $v$ такой, что $v^{*} v \leqslant q(\varphi), v v^{*} \leqslant c(q(\varphi))-q(\varphi)$ (см., например, [1, гл. III, $\S 1$, лемма 1]). Так как след $\varphi_{q(\varphi)}$ полуконечен, то в $\mathfrak{p}_{\varphi}$ найдется ненулевой подпроектор $p$ проектора $v^{*} v$. Тогда $\varphi(|v p|)=\varphi(p)<\infty$, однако $v p \neq q(\varphi) v p q(\varphi)=0$, тем более, $v p \notin \mathfrak{m}_{\varphi}$, а это противоречит свойству $(\mathrm{x})$.

ЗАмЕчАниЕ 3. Воспользовавшись результатами работы [6], можно показать, что для нормального веса $\varphi$ на алгебре фон Неймана $\mathscr{M}$ условия (i)-(ix) эквивалентны еще ряду условий, например, следующему условию “субаддитивности на решетке $\mathscr{M}^{\text {pr } " ~}$ (см. [6, теорема 4]):

(хі) для любых $p, q \in \mathscr{M}^{\mathrm{pr}}$ выполняется неравенство

$$
\varphi(p \vee q) \leqslant \varphi(p)+\varphi(q) .
$$


ЗАмечАниЕ 4 . Пусть теперь $\varphi$ - положительный функционал на $C^{*}$-алгебре $\mathscr{A}$ такой, что для любых эрмитовых элементов $a_{1}, a_{2}$ из $\mathscr{A}$ выполняется неравенство (*). В этом случае, переходя либо к универсальной обертьвающей алгебре фон Неймана, либо к ГНС представлению, индуцированному $\varphi$, можно показать, что $\varphi$ является следом (ср. с теоремой 1 в [11] и теоремой 5 в [6]). Остается, однако, открытым вопрос, позволяет ли вьполнение $(*)$ для любых эрмитовых элементов $a_{1}, a_{2}$ из $\mathscr{A}$ охарактеризовать следы среди весов на $\mathscr{A}$, удовлетворяюших некоторым естественным условиям, например, плотности $\mathfrak{m}_{\varphi}$ в $\mathscr{A}$ и полунепрерьвности снизу по норме.

Авторы выражают свою признательность А. М. Бикчентаеву за полезные обсуждения.

\section{СПИСОК ЦИТИРОВАННОЙ ЛИТЕРАТУРЫ}

[1] Dixmier J. Les algèbres d'opérateurs dans l'espace Hilbertien (algèbres de von Neumann). $2^{e}$ édition. Paris: Gauthier-Villars, 1969.

[2] Haagerup U. Normal weights on $W^{*}$-algebras // J. Funct. Anal. 1975. V. 19. № 3. P. 302-317.

[3] Pedersen G.K., Takesaki M. The Radon-Nikodym theorem for von Neumann algebras // Acta Math. 1973. V. 130. №1-2. P. 53-87.

[4] Upmeier H. Automorphism groups of Jordan $C^{*}$-algebras // Math. Z. 1981. V. 176. № 1. P. 21-34.

[5] Аюпов Ш. А. Классификация и представление упорядоченных йордановых алгебр. Ташкент: ФАН, 1986.

[6] Petz D., Zemánek J. Characterizations of the trace // Linear Algebra Appl. 1988. V. 111. P. 43-52.

[7] Комб Ф. Веса и условные ожидания на алгебрах фон Неймана // Матем. (Сб. переводов). 1974. T. 18. №6. C. $80-113$.

[8] Pedersen G. K. The trace in semi-finite von Neumann algebras // Math. Scand. 1975. V. 37. № 1. P. 142-144.

[9] Шерстнев А. Н. Каждый гладкий вес является $l$-весом // Изв. вузов. Матем. 1977. № 8 . C. $88-91$.

[10] Трунов Н. В., Шерстнев А. Н. Введение в теорию некоммутативного интегрирования // Современные проблемы математики. Новейшие достижения. Итоги науки и техники. Т. 27. М.: ВИНИТИ, 1985. С. 167-190.

[11] Gardner L. T. An inequality characterizes the trace // Canad. J. Math. 1979. V. 31. №6. P. $1322-1328$.

[12] Трунов Н. В. Локально конечные веса на алгебрах Неймана. Казань: Казанский ун-т, 1978. Деп. ВИНИТИ (10 января 1979г.) № 101-79.

[13] Трунов Н.В. К теории нормальных весов на алгебрах Неймана // Изв. вузов. Матем. 1982. № 8. C. 61-70.

[14] Трунов Н.В. Локально конечные веса на алгебрах Неймана и квадратичные формы // Конструктивная теория функций и функцион. анализ. Вып. 5. Казань: Изд-во Казанского ун-та, 1985. С. 80-94. 